\title{
Basin stability approach for quantifying responses of multistable systems with parameters mismatch
}

\author{
P. Brzeski · M. Lazarek · T. Kapitaniak $\cdot$ J. Kurths $\cdot$ P. Perlikowski
}

Received: 20 January 2016/ Accepted: 7 September 2016/Published online: 10 October 2016

(C) The Author(s) 2016. This article is published with open access at Springerlink.com

\begin{abstract}
In this paper we propose a new method to detect and classify coexisting solutions in nonlinear systems. We focus on mechanical and structural systems where we usually avoid multistability for safety and reliability. We want to be sure that in the given range of parameters and initial conditions the expected solution is the only possible or at least has dominant basin of attraction. We propose an algorithm to estimate the probability of reaching the solution in given (accessible) ranges of initial conditions and parameters. We use a modified method of basin stability (Menck et al. in Nat Phys 9(2):89-92, 2013).
\end{abstract}

P. Brzeski · M. Lazarek · T. Kapitaniak ·

P. Perlikowski ( $\square)$

Division of Dynamics, Lodz University of Technology, 90-924 Lodz, Poland

e-mail: przemyslaw.perlikowski@p.lodz.pl

J. Kurths

Potsdam Institute for Climate Impact Research,

14415 Potsdam, Germany

\section{J. Kurths}

Institute of Physics, Humboldt University of Berlin, 12489 Berlin, Germany

\section{J. Kurths}

Department of Control Theory, Nizhny Novgorod State University, Nizhny Novgorod, Russia 603950

\section{J. Kurths}

Institute for Complex Systems and Mathematical Biology, University of Aberdeen, Aberdeen AB24 3UE, UK
In our investigation we examine three different systems: a Duffing oscillator with a tuned mass absorber, a bilinear impacting oscillator and a beam with attached rotating pendula. We present the results that prove the usefulness of the proposed algorithm and highlight its strengths in comparison with classical analysis of nonlinear systems (analytical solutions, path-following, basin of attraction ect.). We show that with relatively small computational effort (comparing to classical analysis) we can predict the behaviour of the system and select the ranges in parameter's space where the system behaves in a presumed way. The method can be used in all types of nonlinear complex systems.

Keywords Basin stability · Multistable systems · Identification of solutions.

\section{Introduction}

In mechanical and structural systems the knowledge of all possible solutions is crucial for safety and reliability. In devices modelled by linear ordinary differential equations we can predict the existing solutions using analytical methods [25, 29]. However, in case of complex, nonlinear systems analytical methods do not give the full view of system's dynamics [3, 20, 24, 31]. Due to nonlinearity, for the same set of parameters more then one stable solution may exist $[5,11,16$, $18,22,23,32]$. This phenomenon is called 
multistability and has been widely investigated in all types of dynamical systems (mechanical, electrical, biological, neurobiological, climate and many more). The number of coexisting solutions strongly depends on the type of nonlinearity, the number of degrees of freedom and the type of coupling between the subsystems. Hence, usually the number of solutions vary strongly when values of system's parameters changes.

As an example, we point out the classical tuned mass absorber $[1,2,6,9,12,17,21,30]$. This device is well known and widely used to absorb energy and mitigate unwanted vibrations. However, the best damping ability is achieved in the neighbourhood of the multistability zone [5]. Among all coexisting solutions only one mitigates oscillations effectively. Other solutions may even amplify an amplitude of the base system. So, it is clear that only by analyzing all possible solutions we can make the device robust.

Similarly, in systems with impacts one solution can ensure correct operation of a machine, while others may lead to damage or destruction $[4,7,14,15,28]$. The same phenomena is present in multi-degree of freedom systems where interactions between modes and internal resonances play an important role [8, 10, 19, 26].

Practically, in nonlinear dynamical systems with more then one degree of freedom it is impossible to find all existing solutions without huge effort and using classical methods of analytical and numerical investigation (path-following, numerical integration, basins of attractions), especially in cases when we analyse a wider range of system's parameters and we cannot precisely predict the initial conditions. Moreover, solutions obtained by integration may have meager basins of attraction and it could be hard or even impossible to achieve them in reality. That is why we propose here a new method basing on the idea of basin stability [23]. The classical basin stability method is based on the idea of Bernoulli trials, i.e., equations of system's motion are integrated $N$ times for randomly chosen initial conditions (in each trial they are different). Analyzing the results we asses the stability of each solution. If there exist only one solution the result of all trials is the same. But, if more attractors coexist we can estimate the probability of their occurrence for a chosen set of initial conditions. In mechanical and structural systems we want to be sure that a presumed solution is stable and has the dominant basin of attraction in a given range of system's parameters. Therefore, we build up a basin stability method by drawing values of system's parameters. We take into account the fact that values of parameters are measured or estimated with some finite precision and also that they can slightly vary during normal operation. When investigated system has multiple attractors or their basins of attractions are especially complex (fractal with the low level of compactness) it may be necessary to support proposed method by the classical analysis to investigate the structure of solutions.

The paper is organized as follows. In Sect. 2 we introduce simple models which we use to demonstrate the main idea of our approach. In the next section we present and describe the proposed method. Section 4 includes numerical examples for systems described in Sect. 2. Finally, in Sect. 5 our conclusions are given.

\section{Model of systems}

In this section we present systems that we use to present our method. Two models are taken from our previous papers $[5,13]$ and the third one was described by Pavlovskaia et al. [27]. We deliberately picked models whose dynamics is well described because we can easily evaluate the correctness and efficiency of the method we propose.

\subsection{Tuned mass absorber coupled to a Duffing oscillator}

The first example is a system with a Duffing oscillator and a tuned mass absorber. It was investigated in [5] and is shown in Fig. 1. The main body consists of mass $M$ fixed to the ground with nonlinear spring (hardening characteristic $k_{1}+k_{2} y^{2}$ ) and a viscous damper (damping coefficient $c_{1}$ ). The main mass is forced externally by a harmonic excitation with amplitude $F$ and frequency $\omega$. The absorber is modelled as a mathematical pendulum with length $l$ and mass $m$. A small viscous damping is present in the pivot of the pendulum.

The equations of the system's motion are derived in [5], hence we do not present their dimension form. Based on the following transformation of coordinates and parameters we reach the dimensionless form: $\omega_{1}^{2}=\frac{k_{1}}{M+m}, \omega_{2}^{2}=\frac{g}{l}, a=\frac{m}{M+m}, b=\left(\frac{\omega_{2}}{\omega_{1}}\right)^{2}, \alpha=\frac{k_{2} l^{2}}{(M+m) \omega_{1}^{2}}$, 


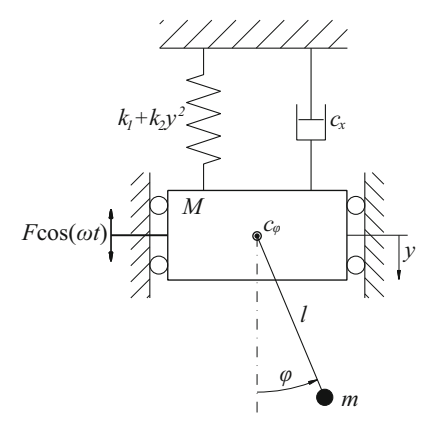

Fig. 1 The model of the first considered system. Externally forced Duffing oscillator with attached pendulum (tuned mass absorber)

$f=\frac{F}{(M+m) l \omega_{1}^{2}}, \quad d_{1}=\frac{c_{x}}{(M+m) \omega_{1}}, \quad d_{2}=\frac{c_{\varphi}}{m l^{2} \omega_{2}}, \quad \mu=\frac{\omega}{\omega_{1}}$, $\tau=t \omega_{1}, x=\frac{y}{l}, \dot{x}=\frac{\dot{y}}{\omega_{1} l}, \ddot{x}=\frac{\ddot{y}}{\omega_{1}^{2} l}, \gamma=\varphi, \dot{\gamma}=\frac{\dot{\varphi}}{\omega_{2}}, \ddot{\gamma}=\frac{\ddot{\varphi}}{\omega_{2}^{2}}$.

The dimensionless equations are as follows:

$\ddot{x}-a b \ddot{\gamma} \sin \gamma-a b \dot{\gamma}^{2} \cos \gamma+x+\alpha x^{3}+d_{1} \dot{x}=f \cos \mu \tau$,

$\ddot{\gamma}-\frac{1}{b} \ddot{x} \sin \gamma+\sin \gamma+d_{2} \dot{\gamma}=0$,

where $\mu$ is the frequency of the external forcing and we consider it as controlling parameter. The dimensionless parameters have the following values: $f=0.5$, $a=0.091, \quad b=3.33, \alpha=0.031, d_{1}=0.132$ and $d_{2}=0.02$. Both subsystems (Duffing oscillator and the pendulum) have a linear resonance for $\mu=1.0$.

\subsection{System with impacts}

As the next example we analyse a system with impacts [27]. It is shown in Fig. 2 and consists of mass $M$ suspended by a linear spring with stiffness $k_{1}$ and a viscous damper with the damping coefficient $c$ to harmonically moving frame. The frame oscillates with amplitude $A$ and frequency $\Omega$. When amplitude of mass $M$ motion reaches the value $g$, we observe soft impacts (spring $k_{2}$ is much stiffer than spring $k_{1}$ ).

The dimensionless equation of motion is as follow (for derivation see [27]) :

$$
\ddot{x}+2 \xi \dot{x}+x+\beta(x-e) \mathrm{H}(x-e)=a \omega^{2} \sin (\omega \tau)
$$

where $x=\frac{y}{y_{0}}$ is the dimensionless vertical displacement of mass $M, \tau=\omega_{n} t$ is the dimensionless time, $\omega_{n}=\frac{k_{1}}{M}, \beta=\frac{k_{2}}{k_{1}}$ the stiffness ratio, $e=\frac{g}{y_{0}}$ the dimensionless gap between equilibrium of mass $M$ and the stop suspended on the spring $k_{2}, a=\frac{A}{y_{0}}$ and $\omega=\frac{\Omega}{\omega_{n}}$ are

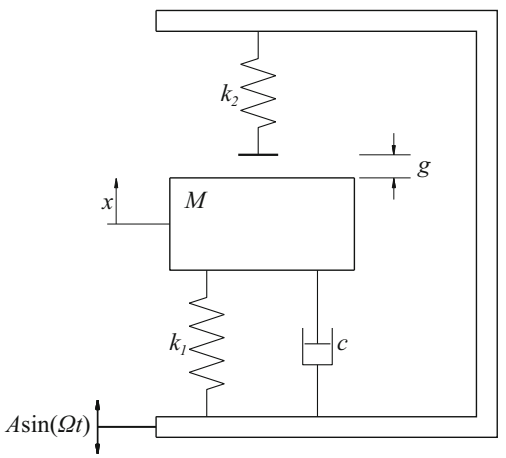

Fig. 2 The model of the second considered system. Externally forced oscillator with impacts

dimensionless amplitude and frequency of excitation, $\xi=\frac{c}{2 m \omega_{n}}$ is the damping ratio, $y_{0}=1.0[\mathrm{~mm}]$ and $\mathrm{H}(\cdot)$ the Heaviside function. In our calculations we take the following values of system's parameters: $a=0.7$, $\xi=0.01, \beta=29, e=1.26$. As a controlling parameter we use the frequency of excitation $\omega$.

\subsection{Beam with suspended rotating pendula}

The last considered system consists of a beam which can move in the horizontal direction and $n$ rotating pendula. The beam has the mass $M$ and supports $n$ rotating, excited pendula. Each pendulum has the same length $l$ and masses $m_{i}(i=1,2, \ldots, n)$. We show the system in Fig. 3 [13]. The rotation of the $i$-th pendulum is given by the variable $\varphi_{i}$ and its motion is damped by the viscous friction described by the damping coefficient $c_{\varphi}$. The forces of inertia of each pendulum acts on the beam causing its motion in the horizontal direction (described by the coordinate $x$ ). The beam is considered as a rigid body, so we do not consider the elastic waves along it. We describe the phenomena which take place far below the resonances for longitudinal oscillations of the beam. The beam is connected to a stationary base by a light spring with the stiffness coefficient $k_{x}$ and viscous damper with a damping coefficient $c_{x}$. The pendula are excited by external torques proportional to their velocities: $N_{0}-\dot{\varphi}_{i} N_{1}$, where $N_{0}$ and $N_{1}$ are constants. If no other external forces act on the pendulum, it rotates with the constant velocity $\omega=N_{0} / N_{1}$. If the system is in a gravitational field (where $g=9.81\left[\mathrm{~m} / \mathrm{s}^{2}\right]$ is the acceleration due to gravity), the weight of the pendulum causes the unevenness of its rotation velocity, i.e., 
Fig. 3 The model of the third considered system. Horizontally moving beam with attached pendulums

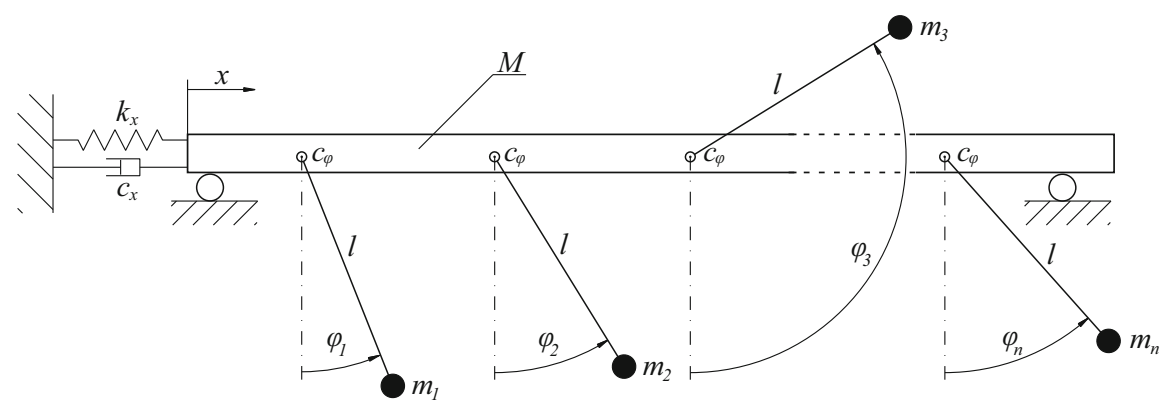

the pendulum slows down when the centre of its mass goes up and accelerates when the centre of its mass goes down.

The system is described by the following set of dimensionless equations:

$m_{i} l^{2} \ddot{\varphi}_{i}+m_{i} \ddot{x} l \cos \varphi_{i}+c_{\varphi} \dot{\varphi}_{i}+m_{i} g l \sin \varphi_{i}=N_{0}-\dot{\varphi}_{i} N_{1}$

$$
\begin{aligned}
& \left(M+\sum_{i=1}^{n} m_{i}\right) \ddot{x}+c_{x} \dot{x}+k_{x} x \\
& =\sum_{i=1}^{n} m_{i} l\left(-\ddot{\varphi}_{i} \cos \varphi_{i}+\dot{\varphi}_{i}^{2} \sin \varphi_{i}\right)
\end{aligned}
$$

In our investigation we analyze two cases: a system with two pendula (where $n=2$ and $i=1,2$ ) and with 20 pendula $(n=20 i=1,2, \ldots, n)$. The values of the parameters are as follows: $m_{i}=\frac{2.00}{n}, \quad l=0.25$, $c_{\varphi}=\frac{0.02}{n}, \quad N_{0}=5.00, \quad N_{1}=0.50, \quad M=6.00$, $g=9.81, \quad c_{x}=\frac{\ln (1.5)}{\pi} \sqrt{k_{x}\left(M+\sum_{i=1}^{n} m_{i}\right)}$ and $k_{x}$ is a controlling parameter. The derivation of the system's equations can be found in [13]. We present the transformation to a dimensionless form in "Appendix".

\section{Methodology}

In [23] Authors present a "basin stability" method which let us estimate the stability and number of solutions for given values of system parameters. The idea behind basin stability is simple, but it is a powerful tool to assess the size of complex basins of attraction in multidimensional systems. For fixed values of system's parameters, $N$ sets of random initial conditions are taken. For each set we check the type of final attractor. Based on this we calculate the chance to reach a given solution and determine the distribution of the probability for all coexisting solutions. This gives us information about the number of stable solutions and the sizes of their basins of attraction.

We consider the dynamical system $\dot{\mathbf{x}}=f(\mathbf{x}, \omega)$, where $\mathbf{x} \in \mathbb{R}^{\mathrm{n}}$ and $\omega \in \mathbb{R}$ is the system's parameter. Let $\mathcal{B} \subset \mathbb{R}^{\mathrm{n}}$ be a set of all possible initial conditions and $\mathcal{C} \subset \mathbb{R}$ a set of accessible values of system's parameter. Let us assume that an attractor $\mathcal{A}$ exists for $\omega \in \mathcal{C}_{\mathcal{A}} \subset \mathcal{C}$ and has a basin of attraction $\beta(\mathcal{A})$. Assuming random initial conditions the probability that the system will reach attractor $\mathcal{A}$ is given by $p(\mathcal{A})$. It is calculated based on classical definition of probability: $p(\mathcal{A})=N(\mathcal{A}) / N$, where $N(\mathcal{A})$ is number of trials leading to attractor $\mathcal{A}$. If this probability is equal to $p(\mathcal{A})=1.0$ this means that the considered solution is the only one in the taken range of initial conditions and given values of parameters. Otherwise other attractors coexist. The initial conditions of the system are random from set $\mathcal{B}_{\mathcal{A}} \subset \mathcal{B}$. We can consider two possible ways to select this set.

1. The first way ensures that the set $\mathcal{B}_{\mathcal{A}}$ includes values of initial conditions leading to all possible solutions. This approach is appropriate if we want to get a general overview of the system's dynamics.

2. In the second approach we use a narrowed set of initial conditions that corresponds to practically accessible initial states.

In our method we chose the second approach because it let us take into account constrains imposed on the system and because in engineering we usually know or expect the initial state of the system with 
some finite precision. The definition of sets of initial conditions is crucial for correctness of obtained results. It should base on brief knowledge of systems dynamics (maximum amplitudes of displacements and velocities of solutions) and information about ranges of accessible initial conditions (maximum values of displacements and velocities that can be applied to the system). In proposed method we are interested in solutions which have basins of attractions in the accessible ranges of initial conditions.

In the classical approach of Menck et al. [23] the values of system's parameters are fixed and do not change during calculations. The novelty of our method is that we not only draw initial conditions but also values of some selected parameters of the system. We assume that the initial conditions and some of the system's parameters are chosen randomly. Then using $N$ trials of numerical simulations we estimate the probability that the system will reach a given attractor $\mathcal{A}(p(\mathcal{A}))$. The idea is to take into consideration the fact that the values of system's parameters are measured or estimated with some finite accuracy which is often hard to determine. Moreover values of parameters can vary during normal operation. Therefore drawing values of parameters we can describe how a mismatch in their values influences the dynamics of the system and estimate the risk of failure. In many practical applications one is interested in reaching only one presumed solution $\mathcal{A}$, and the precise description of other coexisting attractors is not necessary. We usually want to know the probability of reaching the expected solution $p(\mathcal{A})$ and the chance that the system behave differently. If $p(\mathcal{A})$ is sufficiently large, we can treat the other attractors as an element of failure risk.

In our approach we perform the following steps:

1. We pick values of system's parameters from the set $\mathcal{C}_{\mathcal{A}} \subset \mathcal{C}$.

2. We select the $\operatorname{set} \mathcal{C}_{\mathcal{A}}$ so that it consists of all practically accessible values of system's parameters $\omega$. This let us ensure that a given solution indeed exists in a practically accessible range (taking into account the mismatch in parameters).

3. We subdivide the set $C_{A}$ in to $m=1,2, \ldots M$ equally spaced subsets. The subsets $\mathcal{C}_{A}^{m}$ do not overlap and the relation $\bigcup_{m=1 \ldots M} \mathcal{C}_{A}^{m}=C_{A}$ is always fulfilled.
4. Then for each subset $\mathcal{C}_{A}^{m}$ we randomly pick $N$ sets of initial conditions and value of the considered parameter. For each set we check the final attractor of the system.

5. After a suficient number of trials we calculate the probability of reaching a presumed solution or solutions.

6. Finally we describe the relation between the value of the system's parameter and the "basin stability" of reachable solutions....

In our calculations for each range of parameter values (subset $\mathcal{C}_{A}^{m}$ ) we draw from $N=100$ up to $N=1000$ sets of initial conditions and parameter. The value of $N$ strongly depends on the complexity of the analysed system. Also the computation time for a single trial should be adjusted for each system independently such that it can reach the final attractor. In general, we recommend that in most cases $N$ should be at least 100 . If the basins of attractions are especially complex (fractal with low level of compactness) one should take large number of trials $N$ and in the selected range of operation analyze system with classical methods. Nevertheless, presented algorithm in very efficient to select ranges with the low number of co-existing solutions even in extremely complex systems.

\section{Numerical results}

\subsection{Tuned mass absorber coupled to a Duffing oscillator}

At the beginning we want to recall the results we present in our previous paper [5]. As a a summary we show Fig. 4 with a two dimensional bifurcation diagram obtained by the path-following method. It gives bifurcations for varying amplitude $f$ and frequency $\mu$ of the external excitation (see Eq. 1). Lines shown in the plot correspond to different types of bifurcations (period doubling, symmetry breaking, Neimark-Sacker and resonance tongues). We present these lines in one style because the structure is too complex to follow bifurcation scenarios and we do not need that data (details are shown in [5]). We mark areas where we observe the existence of one solution (black colour), or the coexistence of two (grey) and three (hatched area) stable solutions. The remaining part of the diagram (white area) corresponds to 


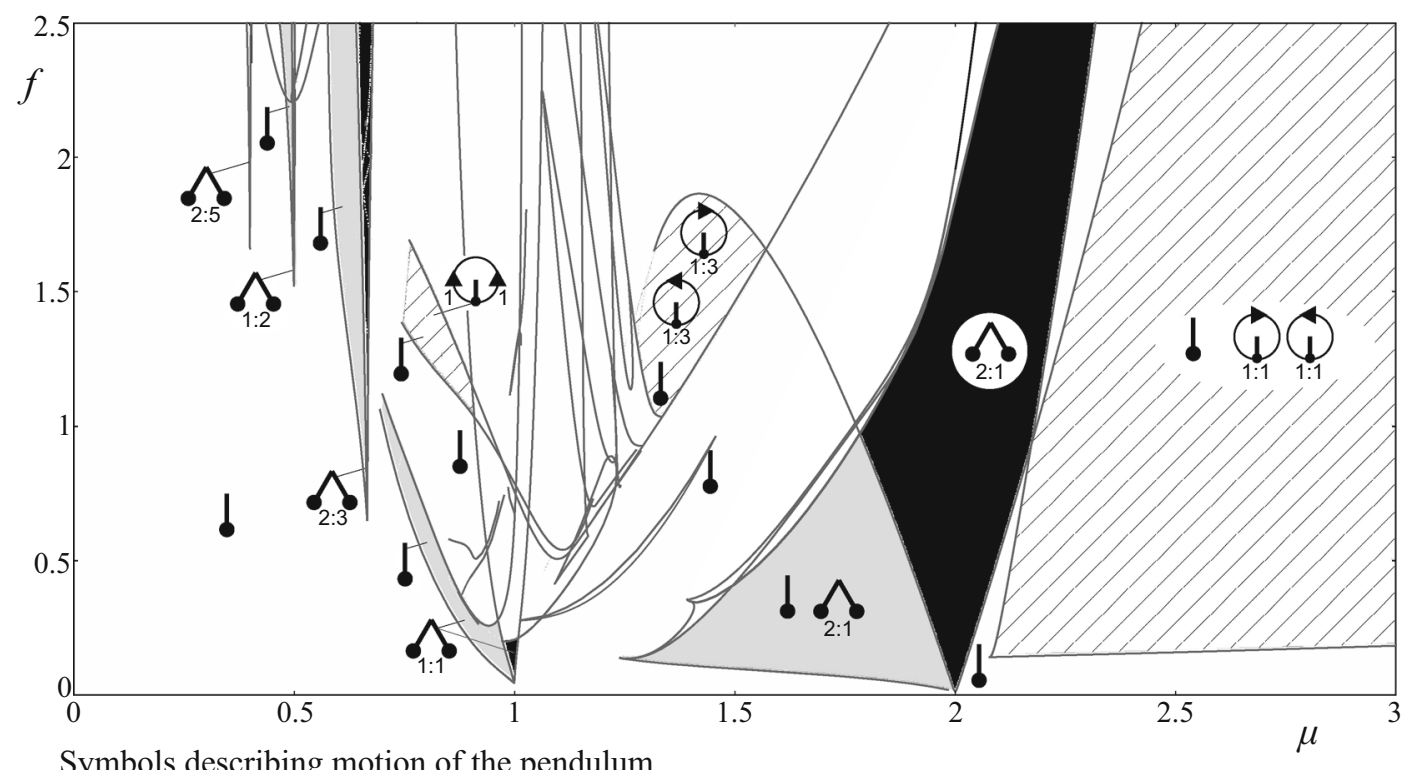

Symbols describing motion of the pendulum
\ - hanging down, no motion
$\widehat{m}_{n}$ - oscillations, resonance $\mathrm{m}$ to $\mathrm{n}$
m:n - one rotation clockwise, one counterclockwise
- rotations resonance, $m$ to $n$

Fig. 4 Two-parameter bifurcations diagram of the system (1) in the plane $(f, \mu)$ showing periodic oscillations and rotations of the pendulum. Black colour indicates one attractor, grey colour shows two coexisting attractors (the same as for black but with a

situations where there are four or more solutions. Additionally, by white colour we also mark areas where only the Duffing system is oscillating in 1:1 resonance with the frequency of excitation and the pendulum is in a stable equilibrium position, i.e., HDP (hanging down pendulum) state. In this case the dynamics of the system is reduced to the oscillations of summary mass $(M+m)$.

The detailed analysis of system 1 is time consuming and creation of Fig. 4 was preceded by complex analysis done with large computational effort. Additionally, the obtained results give us no information about the size of the basins of attraction of each solution-which practically means that some of the solutions may occur only very rarely in the real system (i.e. due to not accessible initial conditions). Nevertheless, such analysis gives us an in-depth knowledge about the bifurcation structure of the system. As we can see, the range where less then three solutions exist is rather small, especially for $\mu<2$.0. To illustrate our method of analysis, we focus on three solutions: 2:1 oscillating resonance, HDP and coexisting stable steady state of the pendulum). In the hatched area we observe the coexistence of stable rotations and a stable steady state of the pendulum. A detailed analysis is presented in [5]

1:1 rotating resonance assuming that only they have some practical meaning.

To show our results obtained with integration, we compute bifurcation diagrams for $f=0.5$ in the range $\mu \in[0.1,3.0]$ (see Fig. 5). In Fig. 5a we increase $\mu$ from 0.1 to 3.0 and in Fig. $5 \mathrm{~b}$ we decrease $\mu$ from 3.0 to 0.1 . As the initial conditions we take the equilibrium position $\left(x_{0}=\dot{x}_{0}=0.0\right.$ and $\left.\gamma_{0}=\dot{\gamma}_{0}=0.0\right)$. In both panels we plot the amplitude of the pendulum $\gamma$. Ranges where the diagrams differ we mark by grey rectangles. It is easy to see that there are two dominating solutions: HDP and 2:1 internal resonance. Near $\mu=1.0$ we observe a narrow range of $1: 1$ and 9:9 resonances and chaotic motion (for details see Figure 6 in [5]). Based on previous results we know that we detected all solutions existing in the considered range, however we do not have information about the size of their basins of attraction and coexistence. Hence the analysis with the proposed method should give us new important information about the system's dynamics. Contrary to the bifurcation diagram obtained by path-following in Fig. 5, we do not 
(a)

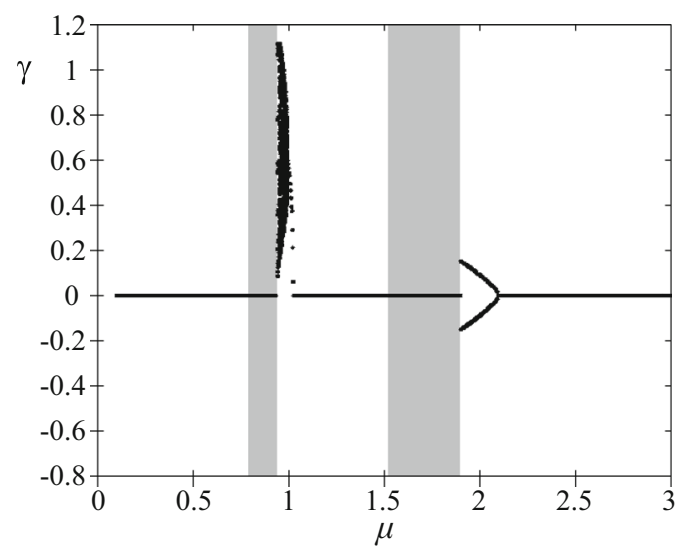

Fig. 5 Bifurcation diagram showing the behaviour of the pendulum suspended on the Duffing oscillator. For subplot a the value of the bifurcation parameter $\mu$ was increased, while for

observe rotating solutions (the other set of initial conditions should be taken).

In Fig. 6 we show the probability of reaching the three aforementioned solutions obtained using the proposed method. The initial conditions are random numbers drawn from the following ranges: $x_{0} \in[-2,2], \quad \dot{x}_{0} \in[-2,2], \quad \gamma_{0} \in[-\pi, \pi]$ and $\dot{\gamma}_{0} \in$ $[-2.0,2.0]$ (ranges there selected basing on the results from [5]). The frequency of excitation is within a range $\mu \in[0,3.0]$ (Fig. 6a, c), then we refine it to $\mu \in$ $[1.25,2.75]$ (Fig. 6b, d). In both cases we take 15 equally spaced subsets of $\mu$ and in each subset we calculate the probability of reaching a given solution. For each subset we calculate 1000 trials each time drawing initial conditions of the system and a value of $\mu$ from the appropriate range. Then we plot the dot in the middle of the subset which indicate the probability of reaching a given solution in each considered range. Lines that connect the dots are shown just to ephasize the tendency. For each range we take $N=1000$ because we want to estimate the probability of a solution with small basin of stability (1:1 rotating periodic solution).

As we can see in Fig. 4, the 2:1 resonance solution exists in the area marked by black colour around $\mu=$ 2.0 and coexists with HDP in the neighbouring grey zone. In Fig. 6 we mark the probability of reaching the 2:1 resonance using blue dots. As we expected, for $\mu<1.4$ and $\mu>2.2$ the solution does not exist. In the range $\mu \in[1.4,2.2]$ the maximum value of probability $p(2: 1)=0.971$ is reached in the subset $\mu \in[1.8,2.0]$ (b)

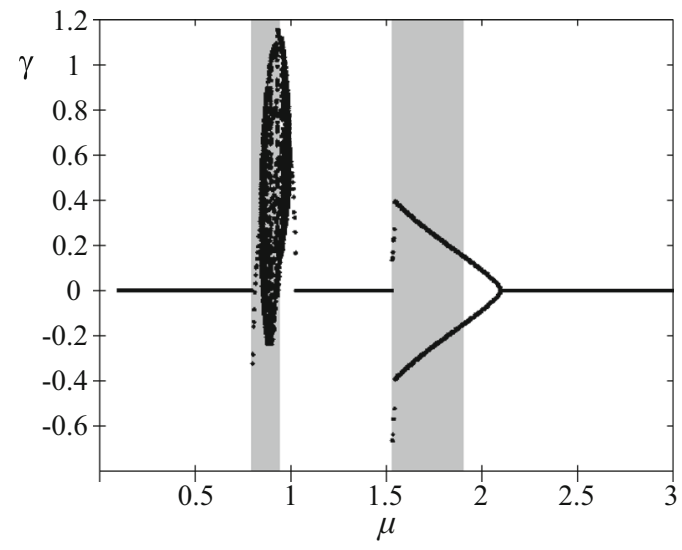

subplot $\mathbf{b}$ we decreased the value of $\mu$. Gray rectangles mark the range of the bifurcation parameter $\mu$ for which different attractors coexist. A detailed analysis is presented in [5]

and outside that range the probability decreases. To check if we can reach $p(2: 1)=1.0$, we decrease the range of parameter's values to $\mu \in[1.25,2.75]$ and the size of subset to $\Delta \mu=0.1$ (we still have 15 equally spaced subsets). The results are shown in Fig. $6 \mathrm{~b}$ similarly in blue colour. In the range $\mu \in[1.95,2.05]$ the probability $p(2: 1)$ is equal to unity and in the range $\mu \in[1.85,1.95]$ it is slightly smaller $p(2: 1)=0.992$. Hence, for both subsets we can be nearly sure that the system reaches the $2: 1$ solution. This gives us indication of how precise we have to set the value of $\mu$ to be sure that the system will behave in a presumed way.

A similar analysis is performed for HDP. The values of probability is indicated by the red dots. As one can see for $\mu<0.8, \quad \mu \in[1.2,1.4]$ and $\mu \in[2.6,2.8]$, the HDP is the only existing solution. The rapid decrease close to $\mu \approx 1.0$ indicates the $1: 1$ resonance and the presence of other coexisting solutions in this range (see [5]). In the range $\mu \in[1.2,1.4]$ the probability $p(\mathrm{HDP})=1.0$ which corresponds to a border between solutions born from 1:1 and 2:1 resonance. Hence, up to $\mu=2.0$ the probability of the HDP solution is a mirror refection of $p(2: 1)$. The same tendency is observed in the narrowed range as presented in Fig. 6b. Finally, for $\mu>2.0$ the third considered solution comes in and we start to observe an increase of probability of the rotating solution $S(\mu, \mathrm{HDP})$ as shown in Fig. 6c. However, the chance of reaching the rotating solution remains small and never exceeds $p(1: 1)=8 \times 10^{-2}$. We also plot the 
(a)

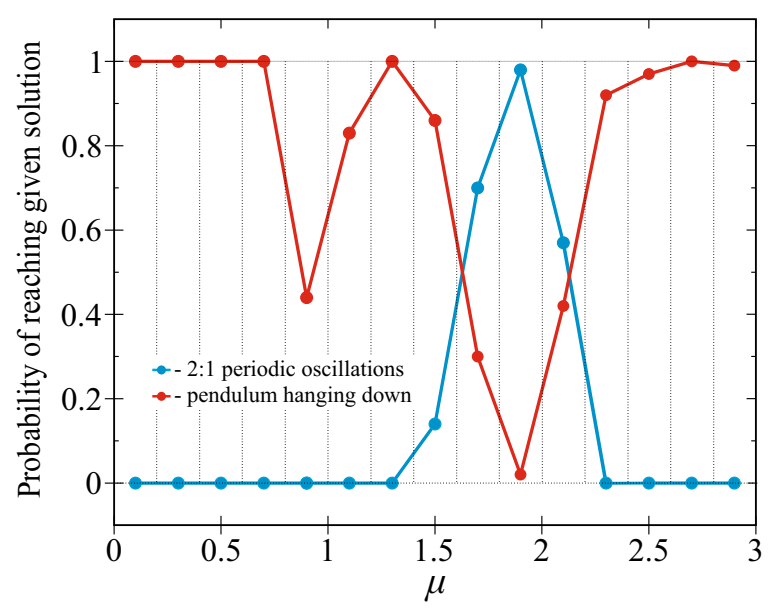

(b)

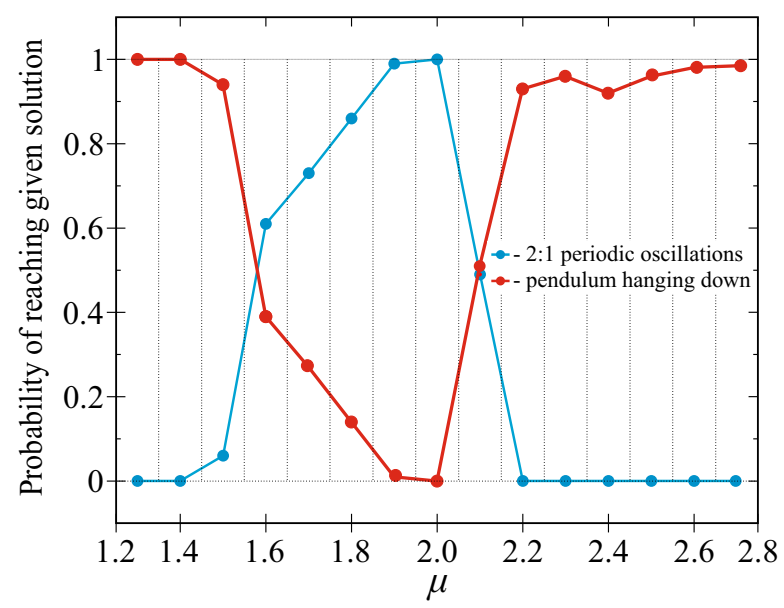

Fig. 6 Probability of reaching given solutions in (1) system with tuned mass absorber. Subplots a, b present solutions with 2:1 periodic oscillations (blue) and without motion of the pendulum (red). Subplots c, d present the probability of

probability of reaching the rotating solution in the narrower range of $\mu$ in Fig. $6 \mathrm{~d}$. The probability is similar to the one presented in Fig. $6 \mathrm{c}-$ it is low and does not exceed $p(1: 1)=8 \times 10^{-2}$. Note that the results presented in Fig. 6a-d are computed for different sets of random initial conditions and parameter values; hence the obtained probability can be slightly different.

\subsection{System with impacts}

In this subsection we present our analysis of different periodic solutions in the system with impacts. A (c)

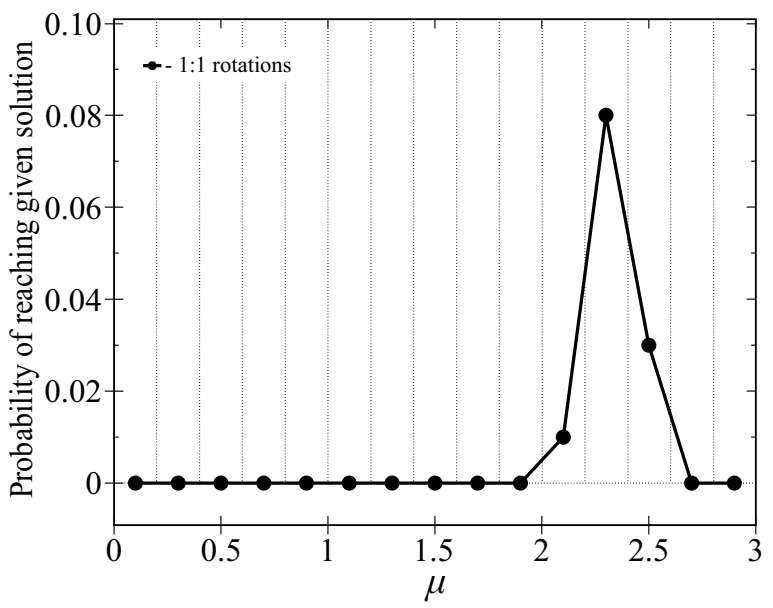

(d)

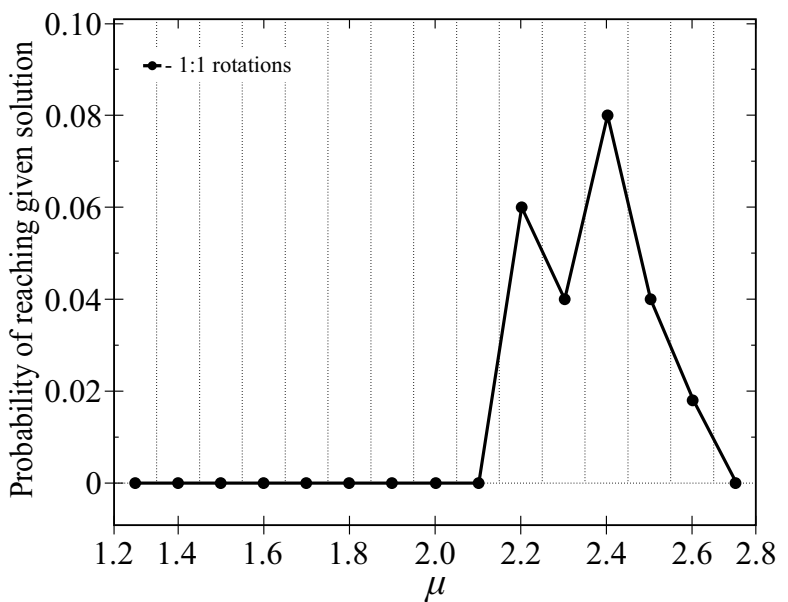

reaching 1:1 rotations (black). (Please note that in both cases (a, b) and (c, d) the initial conditions and parameter are somehow random, hence the results may slightly differ). (Color figure online)

discontinuity usually increases the number of coexisting solutions. Hence, in the considered system we observe a large number of different stable orbits and their classification is necessary. In Fig. 7 we show two bifurcation diagrams with $\omega$ as controlling parameter. Both of them start with initial conditions $x_{0}=0.0$ and $\dot{x}_{0}=0.0$. In panel (a) we increase $\omega$ from 0.801 to 0.8075 ; while in panel (b) we decrease $\omega$ in the same range. We select the range of $\omega$ basing on the results presented in [27]. As one can see, both diagrams differ in two zones marked by grey colour. Hence, we observe a coexistence of different solutions, i.e., in the range $\omega \in[0.8033,0.8044]$ solutions with period-3 
(a)

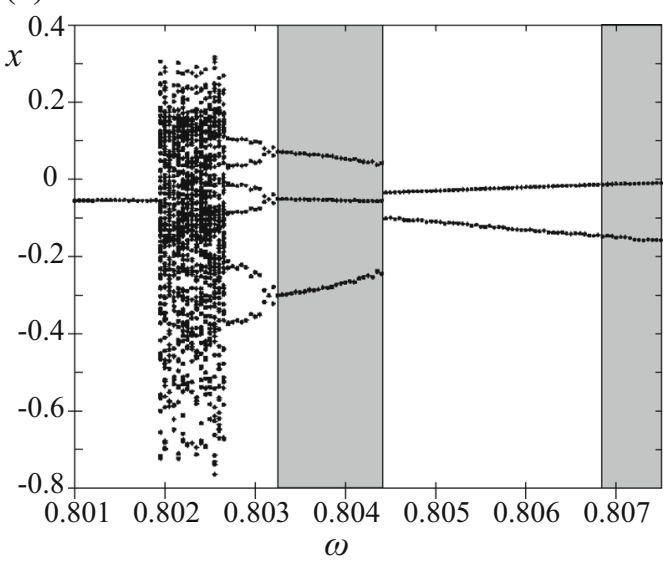

Fig. 7 Bifurcation diagram showing the behaviour of impacting oscillator (2). For subplot a the value of the bifurcation parameter $\omega$ was increased while for subplot $\mathbf{b}$ we decreased

and -2 are present, while in the range $\omega \in$ $[0.8068,0.8075]$ we detected solutions with period-2 and -5. As presented in [27] some solutions appear from a saddle-node bifurcation and we are not able to detect them with the classical bifurcation diagram. The proposed method solves this problem and shows all existing solutions in the considered range of excitation frequency.

We focus on periodic solutions with periods that are not longer than eight periods of excitation. We observe periodic solutions with higher periods in the narrow range of $\omega$ but the probability that they will occur is very small and we can neglect them. All non-periodic solutions are chaotic (quasiperiodic solutions are not present in this system). The results of our calculations are shown in Fig. 8a, b. We take initial conditions from the following ranges $x_{0} \in[-2,2], \dot{x}_{0} \in[-2,2]$. The controlling parameter $\omega$ is changed from 0.801 to 0.8075 with step $\Delta \omega=0.0005$ in Fig. $8 \mathrm{a}$ and from 0.806 to 0.8075 with the step $\Delta \omega=0.0001$ in Fig. $8 \mathrm{~b}$ (in each subrange of excitation's frequency we pick the exact value of $\omega$ randomly from this subset). The probability of periodic solutions is plotted by lines with different colours and markers. We detect the following solutions: period-1, $-2,-3,-5$ (two different attractors with large and small amplitude), -6 and -8 . The dot lines indicate the sum of all periodic solutions' probability (also with period higher then eight). Hence, when its value is below 1 , chaotic solution exist. Dots are drawn for mean value i.e, middle of the (b)

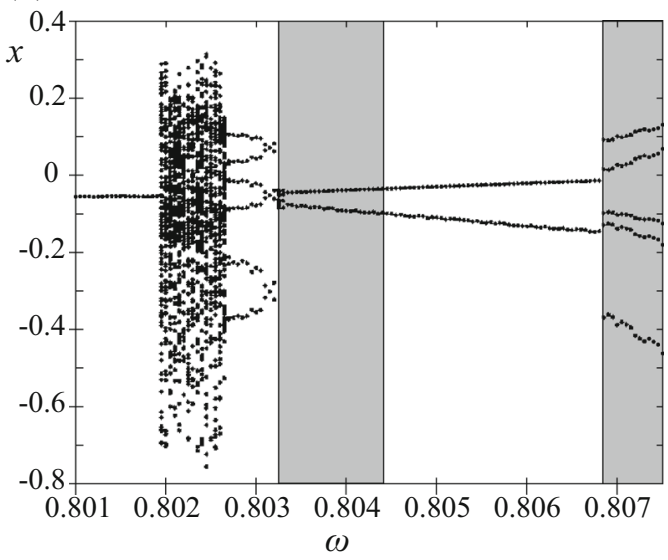

the value of $\omega$. Grey rectangles mark the range of the bifurcation parameter $\omega$ for which different attractors coexist. Further analysis can be found in [27]

subset. For each range we take $N=200$ and we increase the calculation time because the transient time is sufficiently larger than in the previous example due to the piecewise smooth characteristic of spring's stiffness.

As we can see, the chance of reaching a given solution strongly depends on $\omega$. Hence, in the sense of basin stability we can say that stability of solutions rely upon the $\omega$ value. In Fig. 8a the probability of a single solution is always smaller than one. Nevertheless, we observe two dominant solutions: period-5 with large amplitude in the first half of the considered $\omega$ range and period- 2 in the second half of the range. The maximum registered value of probability is $p($ period -2$)=0.92$ and it refers to the period- 2 solution for $\omega \approx 0.80675$. To check if we can achieve even higher probability we analyse a narrower range of $\omega$ and decrease the step (from $\Delta \omega=0.0005$ to $\Delta \omega=0.0001)$. In Fig. $8 \mathrm{~b}$ we see that in range $\omega \in$ $[0.8069,0.807]$ the probability of reaching the period2 solution is equal to 1 . Hence, in the sense of basin stability it is the only stable solution. Also in the range $\omega \in[0.8065,0.8072]$ the probability of reaching this solution is higher then 0.9 and we can say that its basin of attraction is strongly dominant.

Other periodic solutions presented in Fig. 8a are: period- 1 is present in the range $\omega \in[0.801,0.8025]$ with the highest probability $p($ period -1$)=0.4$, period-3 exists in the range $\omega \in[0.803,0.805]$ with the maximum probability $p($ period -3$)=0.36$, 
(a)

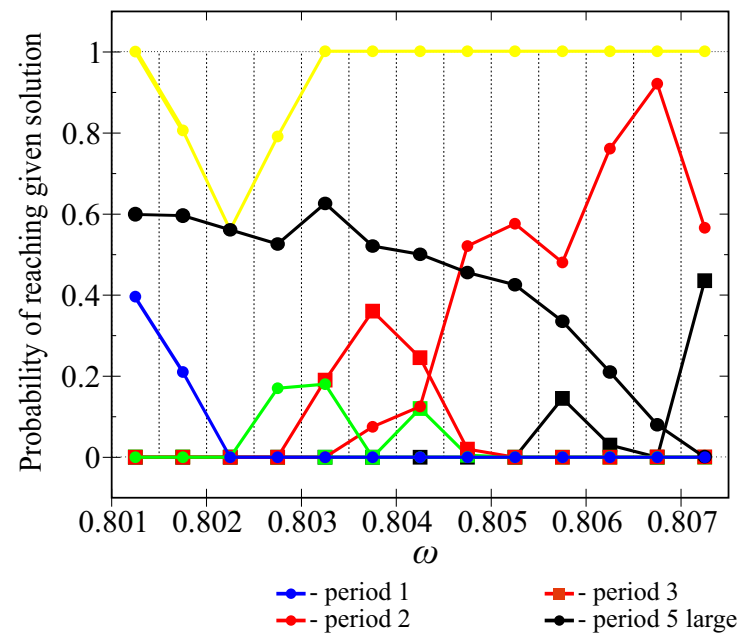

Fig. 8 Probability of reaching given solutions in the impacting system. Subplots a, b present different periodic solutions and the summary probability of reaching any periodic solution. In Subplot a we analyze $\omega \in[0.801,0.8075]$ with the step

period-2 is observed in two ranges $\omega \in$ $[0.8025,0.8035]$ and $\omega \in[0.804,0.8045]$ with the highest probability equal to 0.18 and 0.12 respectively. Solution with period-5 (small amplitude's attractor) exists also in two ranges $\omega \in[0.8055,0.8065]$ and $\omega \in[0.807,0.8075]$ with the highest probability equal to 0.14 and 0.43 respectively.

\subsection{Beam with suspended rotating pendula}

The third considered system consists of a beam that can move horizontally with two $(n=2)$ or twenty $(n=20)$ pendula suspended on it. As a control parameter we use $k_{x}$ which describes the stiffness of the beam's support. For the considered range of $k_{x} \in$ $[100,5000]$ two stable periodic attractors exist in that system. One corresponds to complete synchronization of the rotating pendula. The second one is called antiphase synchronization and refers to the state when the pendula rotate in the same direction but are shifted in phase by $\pi$.

In Fig. 9 we show four bifurcation diagrams with $k_{x}$ as the controlling parameter and a Pioncaré map of rotational speed of the pendula. The subplots $(\mathrm{a}, \mathrm{b})$ refer to the system with two pendula $(n=2)$. We start with zero initial conditions: $x_{0}=0.0, \dot{x}_{0}=0.0$, $\varphi_{10}=0.0, \dot{\varphi}_{10}=0.0, \varphi_{20}=0.0, \dot{\varphi}_{20}=0.0$ and take $k_{x} \in[100,5000]$. The parameter $k_{x}$ is increasing in (b)

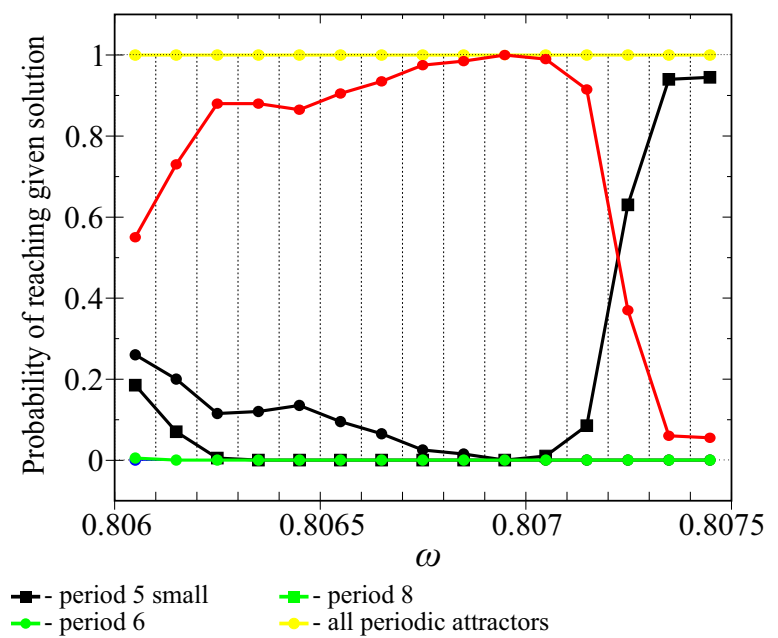

$\Delta \omega=0.0005$, and in subplot $\mathbf{b}$ we narrow the range $\omega \in$ $[0.806,0.8075]$ and decrease the step size $\Delta \omega=0.0001$. (Please note that in cases $(\mathbf{a}, \mathbf{b})$ the initial conditions and parameter are somehow random, hence the results may slightly differ)

subplot (a) and decreasing in (b). We see that in the range marked by grey rectangle both complete and anti-phase synchronization coexist. In subplots (c,d) we present results for twenty pendula $(n=20)$. We start the integration from initial conditions that refer to anti-phase synchronization (two clusters of 10 pendula shifted by $\pi$ ) i.e. $x_{0}=0.1, \dot{x}_{0}=0.00057, \varphi_{k 0}=0.0$, $\dot{\varphi}_{k 0}=9.81, \quad \varphi_{j 0}=3.09, \quad \dot{\varphi}_{j 0}=9.784$ where: $k=$ $1,2, \ldots 10$ and $j=11,12, \ldots 20$. The value of $k_{x}$ is increasing in subplot (c) and decreasing in (d). Similarly as in the two pendula case, we observe the region $\left(k_{x} \in[100,750]\right)$ where two solutions coexist: anti-phase synchronization and non-synchronous state. To further analyse multistability in that system we use proposed method.

In Fig. 10 we present how the probability of reaching a given solution depends on the parameter $k_{x}$. In subplot (a) we show the results for the system with 2 pendula, while in subplot (b) results obtained for the system with 20 pendula suspended on the beam are given. In both cases we consider $k_{x} \in[0,5000]$ and assume the following ranges of initial conditions: $x_{0} \in[-0.15,0.15], \quad \dot{x}_{0} \in[-0.1,0.1], \quad \varphi_{i 0} \in[-\pi, \pi]$, $\varphi_{20} \in[-\pi, \pi], \quad \dot{\varphi}_{10} \in[-3.0,3.0] \quad$ and $\quad \dot{\varphi}_{20} \in$ $[-3.0,3.0]$ in Fig. 10a and $x_{0} \in[-0.15,0.15]$, $\dot{x}_{0} \in[-0.1,0.1], \varphi_{i 0} \in[-\pi, \pi], \dot{\varphi}_{i 0} \in[-\pi, \pi]$ where $i=1 \ldots 20$ in Fig. 10b. We take 20 subsets of parameter $k_{x}$ values with the step equal to $\Delta k_{x}=250$ 


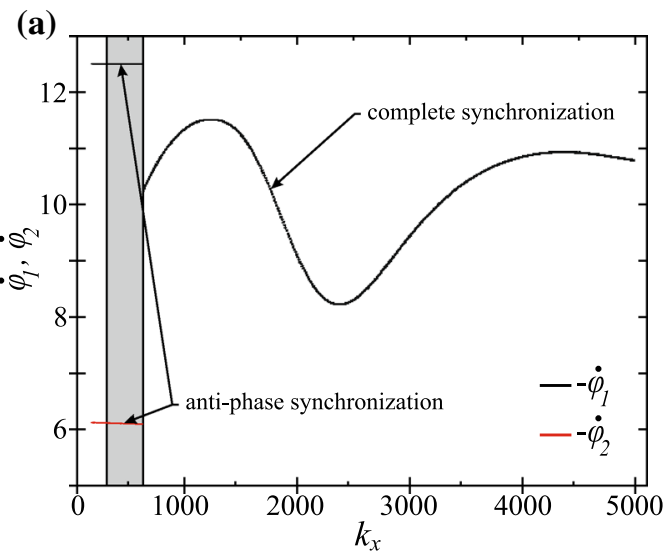

(c)

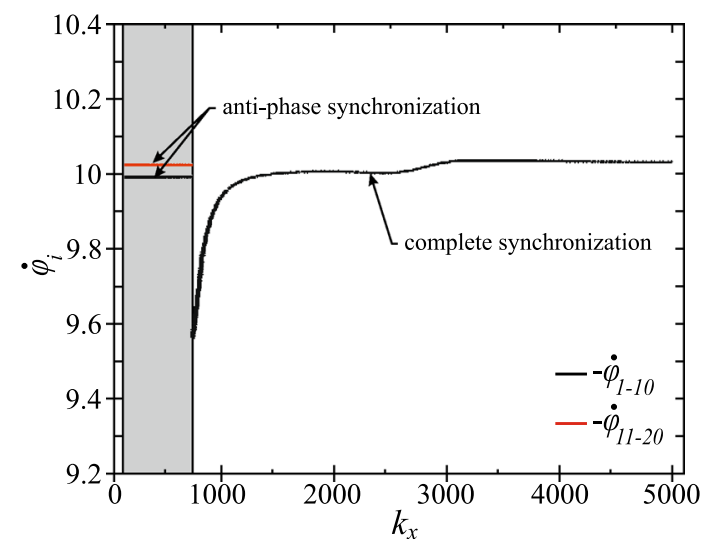

Fig. 9 Bifurctaion diagram showing the behaviour of two (a, b) and twenty (c, d) pendula suspended on the moving beam. For subplots a, $\mathbf{c}$ the value of the bifurcation parameter $k_{x}$ was increased, while for subplots $\mathbf{b}, \mathbf{d}$ we decreased the value of $k_{x}$.

and mark their borders with vertical lines. For each set we run $N=100$ simulations; each one with random initial conditions and $k_{x}$ value drawn from the respective subset. Then, we estimate the probability of reaching given solution. The dots in Fig. 10 indicate the probability of reaching a given solution in the considered range (dots are drawn for mean value, i.e, middle of subset). Contrary to both already presented systems, this one has a much larger dimension of phase space (six and forty two), hence we decide to decrease number of the trials to $N=100$ in order to minimise the time of calculations.

In Fig. 10a we show the results for 2 pendula. When $k_{x} \in[0,250]$ only anti-phase synchronization is possible. Then, with the increase of $k_{x}$ we observe a sudden change in the probability and for $k_{x} \in$

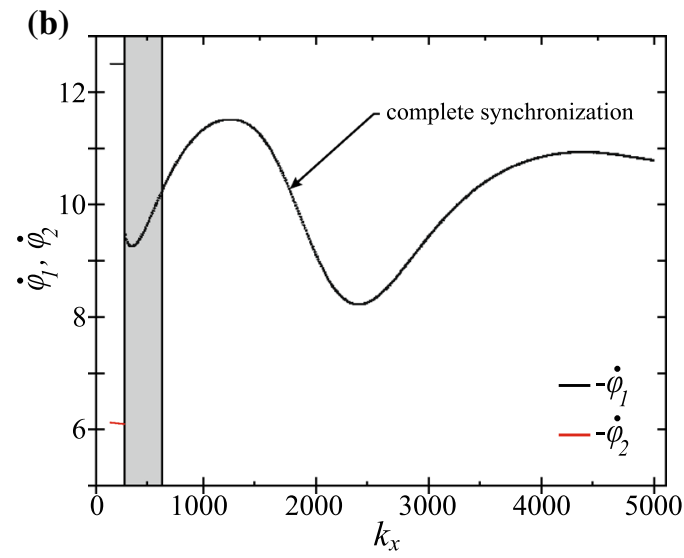

(d)

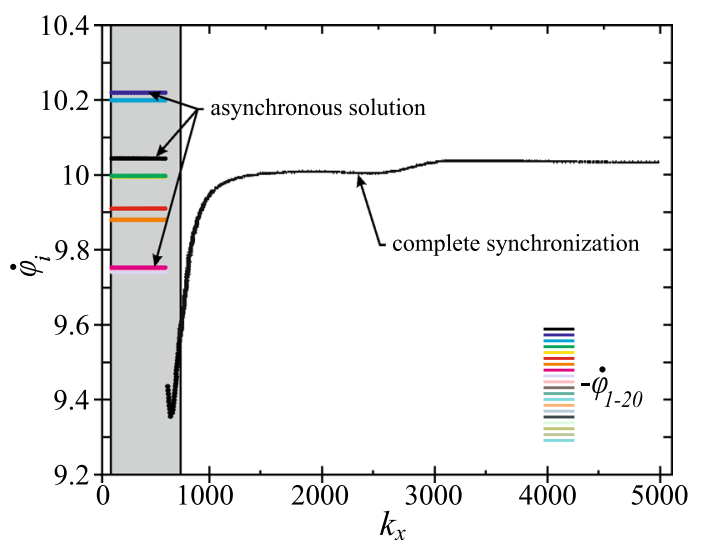

Different colors correspond to Poincaré maps for different pendula. Grey rectangles mark the ranges of the bifurcation parameter $k_{x}$ for which different attractors coexist. Further analysis of number of solutions can be found in [13]

$[750,1750]$ only complete synchronization exists. For $k_{x}>2000$ a probability of reaching both solutions fluctuates around $p$ (complete $)=0.7$ for complete and $p($ anti-phase $)=0.3$ for anti-phase synchronization. Further increase of $k_{x}$ does not introduce any significant changes.

In Fig. 10b we show the results for twenty pendula. For $k_{x} \in[0,250]$ the system reaches solutions different from the two analysed (usually chaotic). Then, the probability of reaching complete synchronization drastically increases and for $k_{x} \in[750,5000]$ it is equal to $p($ complete $)=1.0$ which means that the pendula always synchronize completely. We also present the magnification of the plot where we see that in fact for $k_{x} \in[715,5000]$ we will always observe complete synchronization of the pendula. Please note 
(a)

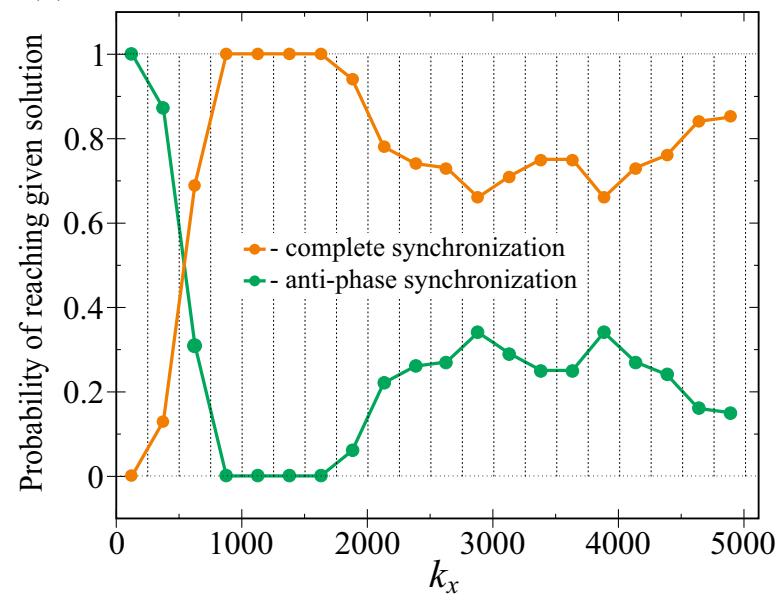

Fig. 10 Probability of reaching given solutions in the system with rotating pendula. Subplot a refers to the case with two pendula and $\mathbf{b}$ with twenty pendula. (Please note that on plot

that for calculating both plots we use random initial conditions and $k_{x}$ value hence, the results for a narrower range may differ. Anti-phase synchronization was never achieved with randomly chosen initial conditions. This means that even though this solution is stable for $k_{x} \in[100,750]$ (see Fig. 9c) it has a much smaller basin of attraction and is extremely hard to obtain in reality. The results presented in Fig. 10 prove that by proper tuning of the parameter $k_{x}$ we can control the systems behaviour even if we can only fix the $k_{x}$ value with finite precision.

\section{Conclusions}

In this paper we propose a new method of detection of solutions' in non-linear mechanical or structural systems. The method allows to get a general view of the system's dynamics and estimate the risk that the system will behave behave differently than assumed. To achieve this goal we extend the method of basin stability [23]. We build up the classical algorithm and draw not only initial conditions but also values of system's parameters. We take this into account because the identification of parameters' values is quite often not very precise. Moreover values of parameters often slowly vary during operation. Whereas in practical applications we usually need certainty that the presumed solution is stable and its basin of stability is large enough to ensure its (b)

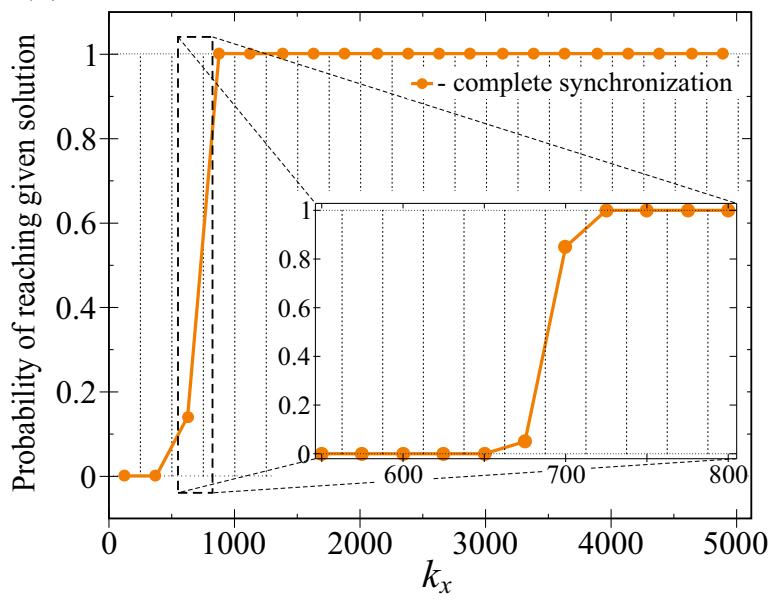

(b) and its magnification the initial conditions and parameter are somehow random, hence the results may slightly differ)

robustness. Hence, there is a need to describe how small changes of parameters' values influence the behaviour of the system. Our method provides such a description and allows us to estimate the required accuracy of parameters values and the risk of unwanted phenomena. Moreover it is relatively time efficient and does not require high computational power.

We show three examples, each for a different class of systems: a tuned mass absorber, a piecewise smooth oscillator and a multi-degree of freedom system. Using the proposed method we can estimate the number of existing solutions, classify them and predict their probability of appearance. Nevertheless, in many cases it is not necessary to distinct all solutions existing in a system but it is enough to focus on an expected solution, while usually other periodic, quasiperiodic and chaotic solutions are classified as undesirable. Such a strategy simplifies the analysis and reduces the computational effort. We can focus only on probable solutions and reduce the number of trials omitting a precise description of solutions with low probability.

The proposed method is robust and can be used not only for mechanical and structural systems but also for any system given by differential equations where the knowledge about existing solutions is crucial.

Acknowledgments This work is funded by the National Science Center Poland based on the decision number DEC2015/16/T/ST8/00516. PB is supported by the Foundation for Polish Science (FNP). 
Open Access This article is distributed under the terms of the Creative Commons Attribution 4.0 International License (http:// creativecommons.org/licenses/by/4.0/), which permits unrestricted use, distribution, and reproduction in any medium, provided you give appropriate credit to the original author(s) and the source, provide a link to the Creative Commons license, and indicate if changes were made.

\section{Appendix}

The motion of the system presented in Fig. 3 is described by the following set of two second order ODEs:

$$
\begin{gathered}
m_{i D} l_{D}^{2} \ddot{\varphi}_{i}^{\prime}+m_{i D} \ddot{x^{\prime}} l_{D} \cos \varphi_{i}^{\prime}+c_{\varphi D} \dot{\varphi}_{i}^{\prime} \\
+m_{i D} g_{D} l_{D} \sin \varphi_{i}^{\prime}=N_{0 D}-\dot{\varphi}_{i}^{\prime} N_{1 D} \\
\left(M_{D}+\sum_{i=1}^{n} m_{i D}\right) \ddot{x^{\prime}}+c_{x D} \dot{x}^{\prime}+k_{x D} x^{\prime} \\
=\sum_{i=1}^{n} m_{i D} l_{D}\left(-\ddot{\varphi}_{i}^{\prime} \cos \varphi_{i}^{\prime}+\dot{\varphi}_{i}^{\prime 2} \sin \varphi_{i}^{\prime}\right)
\end{gathered}
$$

The values of parameters and their dimensions are as follow: $m_{i D}=\frac{2.00}{n}[\mathrm{~kg}], l_{D}=0.25[\mathrm{~m}], c_{\varphi D}=\frac{0.02}{n}[\mathrm{Nms}]$, $N_{0 D}=5.00[\mathrm{Nm}], N_{1 D}=0.50[\mathrm{Nms}], M_{D}=6.00[\mathrm{~kg}]$, $g_{D}=9.81\left[\frac{m}{s^{2}}\right], \quad c_{x_{D}}=\frac{\ln (1.5)}{\pi} \sqrt{k_{x}\left(M+\sum_{i=1}^{n} m_{i}\right)}\left[\frac{N s}{m}\right]$ and $k_{x D}\left[\frac{N}{m}\right]$ is controlling parameter. The derivation of the above equations can be found in [13]. We perform a transformation to a dimensionless form in a way that enables us to hold parameters' values. It is because we want to present new results in a way that thay can be easily compared to results of the investigation presented in [13]. We introduce dimensionless time $\tau=t \omega_{0}$, where $\omega_{0}=1[\mathrm{~Hz}]$, and unit parameters $m_{0}=1.0[\mathrm{~kg}], l_{0}=1.0[\mathrm{~m}]$ and reach the dimensionless equations:

$$
\begin{aligned}
& m_{i} l^{2} \ddot{\varphi}_{i}+m_{i} \ddot{x} l \cos \varphi_{i}+c_{\varphi} \dot{\varphi}_{i}+m_{i} g l \sin \varphi_{i} \\
& \quad=N_{0}-\dot{\varphi}_{i} N_{1} \\
& \left(M+\sum_{i=1}^{n} m_{i}\right) \ddot{x}+c_{x} \dot{x}+k_{x} x \\
& =\sum_{i=1}^{n} m_{i} l\left(-\ddot{\varphi}_{i} \cos \varphi_{i}+\dot{\varphi}_{i}^{2} \sin \varphi_{i}\right)
\end{aligned}
$$

where: $x=\frac{x^{\prime}}{l_{0}}, \dot{x}=\frac{x^{\prime}}{l_{0} \omega_{0}}, \quad \ddot{x}=\frac{\ddot{x^{\prime}}}{l_{0} \omega_{0}^{2}}, \quad \varphi_{i}=\varphi_{i}^{\prime}, \quad \dot{\varphi}_{i}=\frac{\dot{\varphi}_{i}^{\prime}}{\omega_{0}}$, $\ddot{\varphi}_{i}=\frac{\ddot{\varphi}_{i}^{\prime}}{\omega_{0}^{2}}, \quad m_{i}=\frac{m_{i D}}{m_{0}}, \quad l=\frac{l_{D}}{l_{0}}, \quad c_{\varphi}=\frac{c_{\varphi D}}{m_{0} l_{0}^{2} \omega_{0}}, \quad N_{0}=\frac{N_{0 D}}{m_{0} l_{o}^{2} \omega_{0}^{2}}$,
$N_{1}=\frac{N_{1 D}}{m_{0} l_{o}^{2} \omega_{0}}, M=\frac{M_{D}}{m_{0}}, g=\frac{g_{D}}{l_{o} \omega_{0}^{2}}, c_{x}=\frac{c_{x D}}{m_{0} \omega_{0}}$ and dimensionless control parameter $k_{x}=\frac{k_{x D}}{m_{0} \omega_{0}^{2}}$. Dimensionless parameters have the following values: $m_{i}=\frac{2.0}{n}$, $l=0.25, \quad c_{\varphi}=\frac{0.02}{n}, \quad N_{0}=5.0, N_{1}=0.5, \quad M=6.0$, $g=9.81$.

\section{References}

1. Alsuwaiyan AS, Shaw SW (2002) Performance and dynamics stability of general-path centrifugal pendulum vibration absorbers. J Sound Vib 252(5):791-815

2. Arnold FR (1955) Steady-state behavior of systems provided with nonlinear dynamic vibration absorbers. J Appl Math 22:487-492

3. Beléndez A, Hernández A, Márquez A, Beléndez T, Neipp C (2006) Analytical approximations for the period of a nonlinear pendulum. Eur J Phys 27(3):539

4. Blazejczyk-Okolewska B, Kapitaniak T (1998) Co-existing attractors of impact oscillator. Chaos Solitons Fractals 9(8):1439-1443

5. Brzeski P, Perlikowski P, Yanchuk S, Kapitaniak T (2012) The dynamics of the pendulum suspended on the forced Duffing oscillator. J Sound Vib 331:5347-5357

6. Brzeski P, Perlikowski P, Kapitaniak T (2014) Numerical optimization of tuned mass absorbers attached to strongly nonlinear Duffing oscillator. Commun Nonlinear Sci Numer Simul 19(1):298-310

7. Brzeski P, Kapitaniak T, Perlikowski P (2015) Analysis of transitions between different ringing schemes of the church bell. Int J Impact Eng 86:57-66

8. Bux SL, Roberts JW (1986) Non-linear vibratory interactions in systems of coupled beams. J Sound Vib 104(3):497-520

9. Cartmell M, Lawson J (1994) Performance enhancement of an autoparametric vibration absorber by means of computer control. J Sound Vib 177(2):173-195

10. Cartmell MP, Roberts JW (1988) Simultaneous combination resonances in an autoparametrically resonant system. J Sound Vib 123(1):81-101

11. Chudzik A, Perlikowski P, Stefanski A, Kapitaniak T (2011) Multistability and rare attractors in van der Pol-Duffing oscillator. Int J Bifurc Chaos 21(7):1907-1912

12. Chung LL, Wu LY, Lien KH, Chen HH, Huang HH (2013) Optimal design of friction pendulum tuned mass damper with varying friction coefficient. Struct Control Health Monit 20(4):544-559

13. Czolczynski K, Perlikowski P, Stefanski A, Kapitaniak T (2012) Synchronization of slowly rotating pendulums. Int J Bifurc Chaos 22(05):1250128

14. de Souza SLT, Caldas IL (2001) Basins of attraction and transient chaos in a gear-rattling model. J Vib Control 7(6):849-862

15. de Souza SLT, Caldas IL (2004) Controlling chaotic orbits in mechanical systems with impacts. Chaos Solitons Fractals 19(1):171-178 
16. Feudel U, Grebogi C, Poon L, Yorke JA (1998) Dynamical properties of a simple mechanical system with a large number of coexisting periodic attractors. Chaos Solitons Fractals 9(1):171-180

17. Fischer O (2007) Wind-excited vibrations-solution by passive dynamic vibration absorbers of different types. J Wind Eng Ind Aerodyn 95(9):1028-1039

18. Gerson Y, Krylov S, Ilic B, Schreiber D (2012) Design considerations of a large-displacement multistable micro actuator with serially connected bistable elements. Finite Elem Anal Des 49(1):58-69

19. HaQuang N, Mook DT, Plaut RH (1987) Non-linear structural vibrations under combined parametric and external excitations. J Sound Vib 118(2):291-306

20. He J-H (2004) The homotopy perturbation method for nonlinear oscillators with discontinuities. Appl Math Comput 151(1):287-292

21. Ikeda T (2010) Bifurcation phenomena caused by multiple nonlinear vibration absorbers. J Comput Nonlinear Dyn 5:021012

22. Klinshov VV, Nekorkin VI, Kurths J (2016) Stability threshold approach for complex dynamical systems. New J Phys 18:013004

23. Menck PJ, Heitzig J, Marwan N, Kurths J (2013) How basin stability complements the linear-stability paradigm. Nat Phys 9(2):89-92
24. Nayfeh AH (2011) Introduction to perturbation techniques. Wiley, New York

25. Nayfeh AH, Pai PF (2008) Linear and nonlinear structural mechanics. Wiley, New York

26. Orlando D, Gonçalves PB, Rega G, Lenci S (2013) Influence of symmetries and imperfections on the non-linear vibration modes of archetypal structural systems. Int $\mathbf{J}$ Nonlinear Mech 49:175-195

27. Pavlovskaia E, Ing J, Wiercigroch M, Banerjee S (2010) Complex dynamics of bilinear oscillator close to grazing. Int J Bifurc Chaos 20(11):3801-3817

28. Qun-hong L, Qi-shao L (2003) Coexisting periodic orbits in vibro-impacting dynamical systems. Appl Math Mech 24(3):261-273

29. Rao SS, Yap FF (1995) Mechanical vibrations, vol 4. Addison-Wesley Reading, Boston

30. Vazquez-Gonzalez B, Silva-Navarro G (2008) Evaluation of the autoparametric pendulum vibration absorber for a Duffing system. Shock Vib 15:355-368

31. Warminski J, Litak G, Cartmell MP, Khanin R, Wiercigroch M (2003) Approximate analytical solutions for primary chatter in the non-linear metal cutting model. J Sound Vib 259(4):917-933

32. Yanchuk S, Perlikowski P, Popovych OV, Tass PA (2011) Variability of spatio-temporal patterns in non-homogeneous rings of spiking neurons. Chaos 21:047511 